\title{
Erratum to: Polarity-enhanced Gas-Sensing Performance of Au-Loaded ZnO Nanospindles Synthesized via Precipitation and Microwave Irradiation
}

\author{
Authors: Yan Li, * Tan Lv, Fang-Xian Zhao, Xiao-Xue Lian, Yun-Ling Zou, and Qiong Wang \\ Erratum to: Electron. Mater. Lett., Vol. 12, No. 3 (2016), pp. $411-418$ \\ DOI: $10.1007 / \mathbf{s} 13391-016-5391-z$ \\ Correction \\ The original version of this article unfortunately contained an error in the history date. The accepted date \\ of this article is 22 February 2016. The print version of this article was corrected.
}

*Corresponding author: liyan01898@163.com

The online version of the original article can be found at

http://dx.doi.org/10.1007/s13391-016-5391-z.

CKIM and Springer 